\title{
Experimentally constrained density-functional calculations of the amorphous structure of the prototypical phase-change material $\mathrm{Ge}_{2} \mathrm{Sb}_{2} \mathrm{Te}_{5}$
}

\author{
J. Akola, ${ }^{1,2}$ R. O. Jones, ${ }^{1}$ S. Kohara, ${ }^{3,4}$ S. Kimura, ${ }^{3,4}$ K. Kobayashi, ${ }^{5}$ M. Takata, ${ }^{4,6}$ T. Matsunaga, ${ }^{4,7}$ \\ R. Kojima, ${ }^{7}$ and N. Yamada ${ }^{4,7}$ \\ ${ }^{1}$ Institut für Festkörperforschung, Forschungszentrum Jülich, D-52425 Jülich, Germany \\ ${ }^{2}$ Nanoscience Center, Department of Physics, University of Jyväskylä, P.O. Box 35, FI-40014 Jyväskylä, Finland \\ ${ }^{3}$ JASRI/SPring-8, 1-1-1 Kouto, Sayo-gun, Hyogo 679-5198, Japan \\ ${ }^{4} J S T$, CREST, 5 Sanbancho, Chiyoda-ku, Tokyo 102-0075, Japan \\ ${ }^{5}$ Beamline Station at SPring-8, National Institute for Materials Science, 1-1-1 Kouto, Sayo-gun, Hyogo 679-5198, Japan \\ ${ }^{6}$ SPring-8/RIKEN, 1-1-1 Kouto, Sayo-gun, Hyogo 679-5148, Japan \\ ${ }^{7}$ Panasonic Corporation, 3-1-1 Yagumo-Nagamachi, Moriguchi, Osaka 570-8501, Japan
}

(Received 24 April 2009; published 7 July 2009; corrected 15 July 2009)

\begin{abstract}
Phase change materials involve the rapid and reversible transition between nanoscale amorphous $(a-)$ and crystalline $(c-)$ spots in a polycrystalline film and play major roles in the multimedia world, including nonvolatile computer memory. The materials of choice are alloys of $\mathrm{Ge}, \mathrm{Sb}$, and Te, e.g., $\mathrm{Ge}_{2} \mathrm{Sb}_{2} \mathrm{Te}_{5}$ (GST) in digital versatile disk-random access memory. There has been much speculation about the structure of $a$ - GST, but no model has yet received general acceptance. Here we optimize the structure by combining the results of density-functional calculations with high-energy $\mathrm{x}$-ray diffraction data and $\mathrm{x}$-ray photoelectron spectroscopy (XPS) measurements of the electronic densities of states to determine the structure. The structure agrees very well with available experimental data, including the differences in the XPS data between the amorphous and crystalline structures.
\end{abstract}

DOI: 10.1103/PhysRevB.80.020201

PACS number(s): 61.43.Bn, 61.43.Dq, 64.70.Nd, 71.15.Pd

Phase change materials play crucial roles in rewritable media [digital versatile disk (DVD)-rewriteable, DVD-RAM, and Blu-ray Disc] and are poised for wider applications to nonvolatile computer memory. ${ }^{1}$ They involve the rapid and reversible change between nanoscale amorphous $(a-)$ and crystalline $(c-)$ spots in a polycrystalline film, and alloys of $\mathrm{Ge}, \mathrm{Sb}$, and Te have been the materials of choice for over 20 years. ${ }^{2}$ Crystallization is the rate-limiting step in a write/ erase cycle, and this has led to much speculation about the structure of these alloys, particularly $\mathrm{Ge}_{2} \mathrm{Sb}_{2} \mathrm{Te}_{5}$ (GST). A recent reverse Monte Carlo (RMC) analysis of synchrotronradiation high-energy x-ray diffraction (XRD) data from $a$ GST (Ref. 3) indicated that the structure was dominated by four- and six-membered rings also found in the crystal but different from $a$-GeTe in having no Ge-Ge bonds. Extensive density-functional (DF) calculations on liquid and $a$-GST extended this picture: ${ }^{4}$ Medium-to-long-ranged order exists among Te atoms, and cavities and $A B A B$ squares $(A: \mathrm{Ge} / \mathrm{Sb}, B: \mathrm{Te})$ characterize these materials and their phase changes. ${ }^{5}$ Here we use density-functional calculations, high-energy XRD data, and published X-ray photoelectron spectroscopy (XPS) data to refine the amorphous structure of GST.

The dramatic increase in experimental data on GST in recent years has provided an ideal database for testing structural models, but there is little consensus on the structure of $a$-GST. Extended x-ray absorption fine-structure (EXAFS) measurements showed that the Ge-Te bond in $a$-GST (2.61 $\AA$ ) is significantly shorter than in the crystal $(2.83 \AA)$, and the Sb-Te bond is also reduced. ${ }^{6}$ If the Te sublattice is assumed to remain cubic, the $\mathrm{Ge}$ and $\mathrm{Sb}$ atoms must be displaced significantly from their crystalline positions, and the order-disorder transition could occur as an "umbrella" flip of
Ge atoms from octahedral to tetrahedral positions. ${ }^{6}$ This model is, however, not consistent with subsequent DF calculations, ${ }^{7}$ which indicated that the latter would relax without energy barrier to the octahedral structure of $c$-GST. An RMC analysis of electron-diffraction data favored an amorphous structure comprising distorted and misaligned rings. ${ }^{7}$ Other EXAFS measurements on GST found evidence of $\mathrm{Ge}-\mathrm{Ge}$ bonds and overcoordinated Te atoms. ${ }^{8} \mathrm{~A}$ recent RMC study of $a$-GST using EXAFS, neutron-diffraction and X-ray diffraction data ${ }^{9}$ indicated the presence of Ge-Ge and Ge-Sb bonds, which are absent in $c$-GST. The coordination numbers of all three elements were found to obey the " $8-N$ rule," where $N$ is the number of valence electrons. These papers do not discuss the electronic structures of different phases of GST, which are crucial to its role as a phasechange memory material.

An ideal structural model of $a$-GST would:

(i) reproduce the measured $S(Q)$ and the total pair distribution function $g(r)$ for XRD and/or neutron diffraction;

(ii) have bond lengths that are not artificially short and are consistent with EXAFS measurements;

(iii) have an electronic structure with a band gap at the Fermi energy; and

(iv) have a DF total energy close to the minimum.

We seek this goal by combining data from high-energy XRD (Ref. 3) and XPS measurements on $a$ - and $c$-GST (Ref. 10) with DF simulations of a large sample (460 atoms) over hundreds of picoseconds. The original DF calculations ${ }^{4}$ agreed reasonably well with measured $S(Q)$ and $g(r)$ for $\mathrm{GST}^{3}$ but several details were open to improvement. The present collaboration began when we discovered that the structure obtained in the original RMC fit to the XRD measurements ${ }^{3}$ gave rise to a metallic density of states 
(DOS), i.e., no band gap at the Fermi energy. Our strategy is related to the "experimentally constrained molecular relaxation" (ECMR) of Biswas et al. ${ }^{11}$ and combines the strengths of theory and experiment to allow us to approach the above goal.

Our first combined molecular-dynamics (MD)/DF calculations ${ }^{4}$ were performed with the CPMD package, ${ }^{12}$ using the approximation of Perdew, Burke, and Ernzerhof (PBE) (Ref. 13) for the exchange-correlation energy. In this work we use the approximation of Tao et al. (TPSS), ${ }^{14}$ which was developed recently to improve results obtained with generalized gradient approximations, including PBE. The TPSS form has orbital-dependent terms that increase the computing demands, but structures and energy differences are generally better. ${ }^{14}$ The initial structure was based on that of crystalline GST (rock salt) with 460 atoms and 52 vacancies, and the starting temperature was $3000 \mathrm{~K}$ (liquid). The long simulation time (300 ps) is essential for a realistic description of the number of "wrong bonds" (i.e., bonds that do not occur in $c$-GST), whose number decreases steadily as the temperature is lowered. A cutoff distance of $3.2 \AA$ is used to analyze bond angles and ring distributions.

The structures of $c$ - and $a$-GST (Ref. 4) (460 atoms) were reoptimized using the TPSS functional, and the pair distribution function (PDF) for $c$-GST is in excellent agreement with XRD measurements. Both EXAFS and XRD results indicate the presence of the double maximum, and the calculated $\mathrm{Ge}-\mathrm{Te}$ and $\mathrm{Sb}-\mathrm{Te}$ bond distributions have double maxima that were not evident in the PBE results. The shorter Ge-Te and $\mathrm{Sb}$-Te bond lengths (2.84 and $2.93 \AA$, respectively) agree very well with EXAFS values ( 2.83 and $2.91 \AA) .{ }^{6}$ The longer bonds have maxima above $3.1 \AA$. TPSS bonds in $a$-GST are $1-2 \%$ shorter than with the PBE functional, and the coordination numbers (Ge: 3.9, Sb: 3.5, and Te: 2.7) are lower than in Ref. 4 (4.2, 3.7, and 2.9). The cohesive energy is $2.62 \mathrm{eV}$, and $c$-GST is $0.058 \mathrm{eV} /$ atom more stable than a-GST.

The reoptimized $a$-GST geometry was taken as input for RMC refinement using the $\mathrm{RMC}++\operatorname{program}^{15}$ and the experimental x-ray total structure factor $S(Q)$. The maximum movement for any atom at each MC step was $0.14 \AA$. The experimental $S(Q)$ can be reproduced very well with a range of input parameters, but RMC simulations without constraints on the bond angles led to a metallic DOS, even when the initial structure was semiconducting. A semiconducting structure that satisfies the x-ray structural information for $a$ GST can be obtained in practice only by using an iterative scheme where the bond angle distributions Te-Ge-Te, Te-Sb$\mathrm{Te}, \mathrm{Ge}-\mathrm{Te}-\mathrm{Ge}, \mathrm{Ge}-\mathrm{Te}-\mathrm{Sb}$, and $\mathrm{Sb}-\mathrm{Te}-\mathrm{Sb}$ are constrained to values near those found in the DF calculations. Very broad distributions can result otherwise, as found in $a$-Si, where the width of the Si-Si-Si bond angle distribution is closely related to the electronic DOS. ${ }^{16}$ The Ge-Te-Ge and Te-Ge-Te bond angles are very sensitive to the DOS, and the weighting factor for the Ge-Ge correlation in X-ray diffraction experiments is small. The TPSS bond lengths are slightly shorter than PBE values, and the angular distributions are very similar (see Fig. 3 in supplementary material). The use of the PBE approximation in the present scheme instead of TPSS would lead to very similar optimized structures.
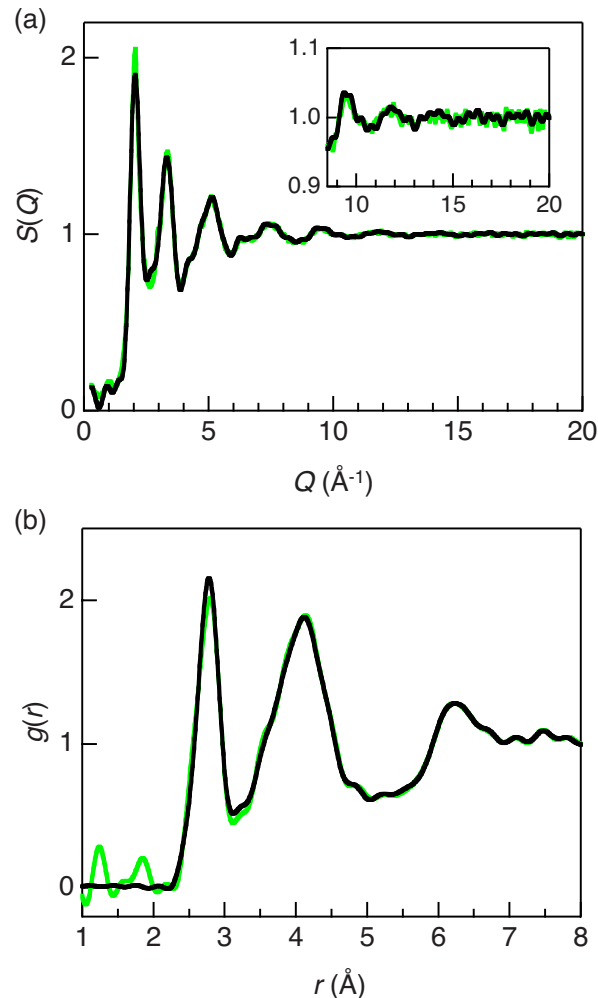

FIG. 1. (Color online) (a) Structure factor $S(Q)$ in $a$-GST. Green (gray): XRD (Ref. 3); black: calculated for RMC-optimized geometry. Inset: data for the high- $Q$ values. (b) Total radial distribution functions (XRD weights).

The RMC optimization of the TPSS structure yields a structure factor $S(Q)$ in excellent agreement with XRD results [Fig. 1(a)], with small differences in amplitude near the first two peaks. The excellent description of the high- $Q$ region is important because the short-range atomic correlations in the XRD pair distribution function $g(r)$ [Fig. 1(b)] is obtained by Fourier transforming $Q[S(Q)-1]$ and is affected strongly by the high- $Q$ portion of $S(Q)$. The calculated $g(r)$ follows the experimental curve almost perfectly with minor deviations near the first peak, and the total energy is only $0.079 \mathrm{eV} /$ atom higher than that of the DF energy minimum. The electronic DOS shows a band gap at the Fermi level.

The partial pair distribution functions $g_{i j}(r)$ obtained from the RMC-optimized structure are shown in Fig. 1 in supplementary material. ${ }^{17}$ The Sb-Te and Ge-Ge bond lengths $(2.85$ and $2.45 \AA$, respectively) agree well with EXAFS measurements. ${ }^{8}$ The main Ge-Te peak is at $2.75 \AA$, but the shoulder at $2.60 \AA$ can be assigned unambiguously to tetrahedrally coordinated $\mathrm{Ge}$ atoms ${ }^{17}$ and agrees well with the measured value $(2.63 \pm 0.01 \AA) .8$ "Wrong bonds" (Ge-Ge, $\mathrm{Ge}-\mathrm{Sb}, \mathrm{Sb}-\mathrm{Sb}$, and Te-Te) occur with small partial coordination numbers $(0.2-0.6)$, and the $A B$ alternation found in $c$ GST is obvious. The total coordination numbers (Ge: 3.9, Sb: 3.4, and Te: 2.6) are slightly lower than for the TPSS starting geometry, particularly in Te, but overcoordination in $\mathrm{Sb}$ and $\mathrm{Te}$ is clear. The EXAFS values are $3.9 \pm 0.8$, $2.8 \pm 0.5$, and $2.4 \pm 0.8 .^{8}$ The dependence of coordination number on cutoff is given in Table I of the supplementary information. 17 

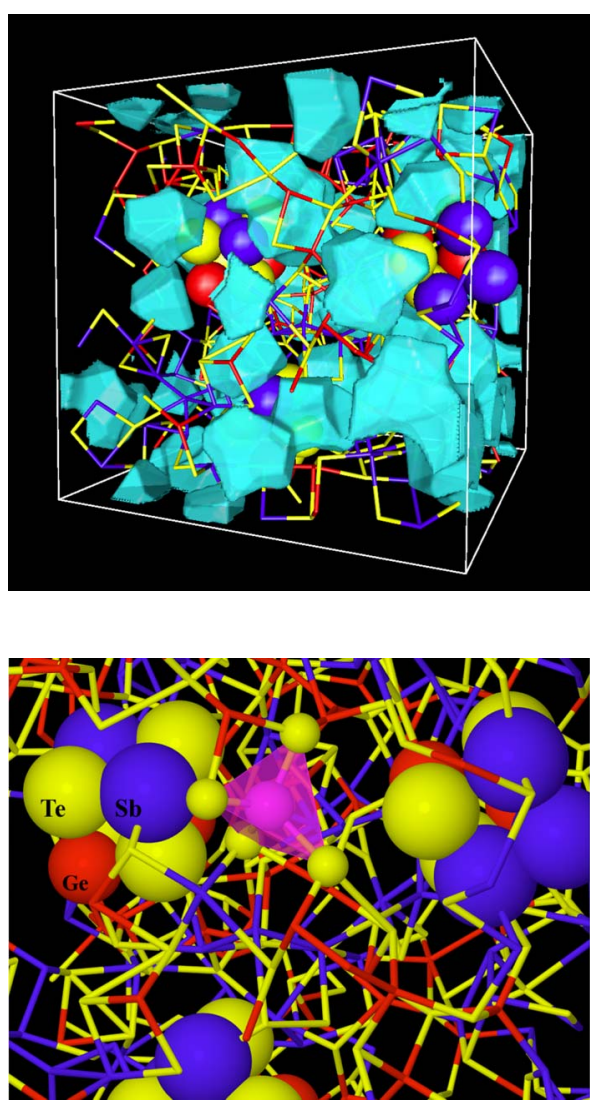

FIG. 2. (Color online) (upper) RMC structure of $a$-GST [red (gray): Ge; blue (dark gray): Sb; and yellow (white): Te]. The 37 cavities are shown in light blue (light gray). (lower) Three cubes in part of optimized structure: two with $A B$ alternation and one with $\mathrm{Sb}-\mathrm{Sb}$ bonds. A tetrahedral Ge-centered structure is shown.

Figure 2 shows the RMC-optimized structure and important components of the amorphous phase, including cavities [see Fig. 2(a)], which make up $13.7 \%$ of the total volume. This is slightly more than in Ref. 4 (as expected from the lower coordination) and higher than the vacancy concentration of $c$ - GST $(10 \%)$. The material comprises cubic subunits [Fig. 2(b)], many of which (including $A B A B$ squares) show perfect $A B$ alternation. These features support the idea of a nucleation-driven phase change, ${ }^{18}$ where cubic subunits act as nucleation centers and cavities provide space for the concerted rearrangement of square units to form a cubic lattice.

Amorphous GST contains many local environments: one $\mathrm{Ge}^{-} \mathrm{Te}_{4}$ tetrahedron is highlighted in Fig. 2(b), and we show some common Ge-centered configurations in Fig. 2 in supplementary material. Ge has both (distorted) octahedral and tetrahedral configurations with different bond lengths ( $41 \%$ of the Ge atoms are "tetrahedral"19), and three- and fivefold Ge configurations occur frequently. As in Ref. 4, we use a cutoff distance of $3.2 \AA$ in evaluating bond angle distributions (see Fig. 3 in supplementary material). Octahedral features can be identified by maxima at 90 and $180^{\circ}$, and tetrahedral coordination is never favored in Sb. The statistics of $n$-membered irreducible rings (closed loops) are shown in Fig. 3. Four-membered rings (over $90 \%$ have the form $A B A B)$ dominate, and there are very few triangles. The lower (a)

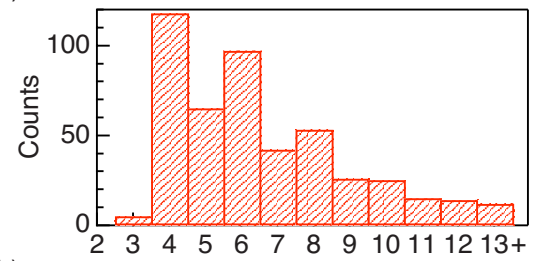

(b)

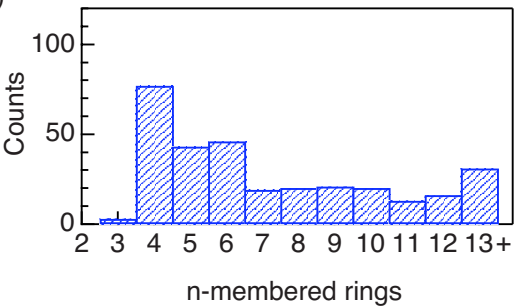

FIG. 3. (Color online) Irreducible rings in $a$ - GST (a) Ref. 4; (b) RMC-optimized geometry. "13+:" rings with more than 12 atoms.

overall coordination means that there are fewer rings than found in Ref. 4, and the odd-even alternation is less pronounced. The increased weight of larger rings correlates with the presence of larger cavities.

Finally we optimize the RMC geometry to find the nearest DF minimum as this is essential for comparing the electronic structures of the $a$ - and $c$-phases. The total energy found is $0.006 \mathrm{eV} /$ atom above the initial DF energy minimum, and the coordination numbers $(3.9,3.5$, and 2.7) are near the RMC values. Some longer bonds lead to a shift in the first peak of the PDF, which affects $S(Q)$ (see Fig. 4 in supplementary material). Both $a$ - GST and $c$ - GST are semiconducting, with band gaps near $0.2 \mathrm{eV}$. The calculated and XPS DOS [Fig. 4(a)] are similar, and the corresponding differences between $a$ - GST and $c$-GST [ $\Delta_{\text {DOS }}$, Fig. 4(b)] agree
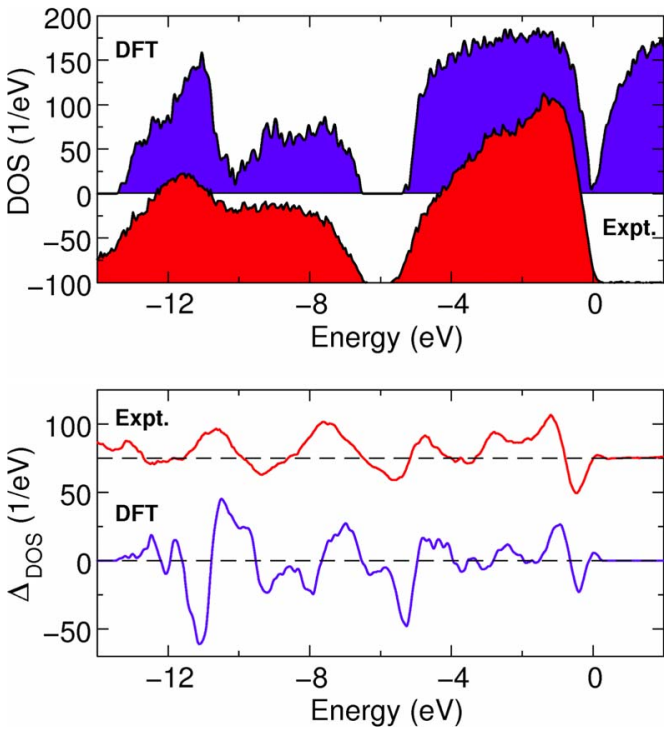

FIG. 4. (Color online) (a) (upper) XPS valence-band spectrum of $a$-GST [red (gray), arbitrary units] (Ref. 10) and the calculated electronic DOS [blue (dark gray), electrons per eV]. (b) (lower) $\Delta_{\text {DOS }}$ (see text). Blue (black): DF calculations, red (dark gray): XPS. Units as in (a). 
remarkably well. The structure we find reproduces the changes in electronic structure occurring during the phase change.

The iterative combination of DF calculations (without adjustable parameters) and RMC fitting leads in amorphous GST to a structure that satisfies our goals. The initial and optimized structures have semiconducting electronic structures, and the TPSS approximation leads to even better bond lengths and angles than PBE values. We have not calculated the EXAFS spectrum, but measured EXAFS bond lengths agree well with our calculated peaks or shoulders. A range of local environments occurs. Octahedral and tetrahedral Ge sites coexist, but an accurate fit to $S(Q)$ can be found without presupposing the existence of either. The coordination numbers in our melt-quench simulations do not satisfy the " $(8$ $-N$ ) rule," which would also require a significant number of "wrong bonds" for Ge and Sb to compensate the Te undercoordination.

In the ECMR approach of Biswas et al., ${ }^{11}$ random structures are modified to incorporate experimental information and used in a two-step process employing RMC methods and DF energy relaxation. Our procedure is related, but there are significant differences. We focus on phase-change materials for which the melt-quench time scale is much shorter than in glassy materials. Moreover, our starting structure resulted from an extensive, self-consistent, and gradual (300 ps) DF optimization of a high-temperature structure and had a band gap at the Fermi energy. The ECMR approach has so far been limited to energy and force calculations using the Harris functional. ${ }^{20}$ This is computationally very efficient, but it is not self-consistent and is an uncontrolled approximation in systems, such as GST, with many vacancies and significant charge transfers between atoms.

The procedure described here has allowed us to refine the picture of $a$-GST that follows the work of Refs. 3-5: (a) ring structures play an essential role in the phase change, and $A B A B$ squares, structural units in $c$-GST, are dominant in $a$ GST; (b) numerous cavities provide space for the phase change to occur without breaking many bonds; and (c) overcoordinated $\mathrm{Sb}$ and Te and a small number of "wrong bonds" indicate that $a$-GST is a poor glass former. These features are keys to the rapid phase change in GST.

The experimental work was supported by the Japan Science and Technology Agency, and the calculations were performed on IBM BlueGene/L, BlueGene/P, and p690 computers in the Forschungszentrum Jülich with grants from the FZ Jülich and the John von Neumann Institute for Computing. R.O.J. thanks SPring-8 and its staff for hospitality and support during a research visit.
${ }^{1}$ M. Wuttig and N. Yamada, Nature Mater. 6, 824 (2007).

${ }^{2}$ N. Yamada, E. Ohno, N. Akahira, K. Nishiuchi, K. Nagata, and M. Takao, Jpn. J. Appl. Phys. 26, 61 (1987).

${ }^{3}$ S. Kohara, K. Kato, S. Kimura, H. Tanaka, T. Usuki, K. Suzuya, H. Tanaka, Y. Moritomo, T. Matsunaga, N. Yamada, Y. Tanaka, H. Suematsu, and M. Takata, Appl. Phys. Lett. 89, 201910 (2006).

${ }^{4}$ J. Akola and R. O. Jones, Phys. Rev. B 76, 235201 (2007); J. Phys.: Condens. Matter 20, 465103 (2008); see also Phys. Rev. Lett. 100, 205502 (2008).

${ }^{5}$ S. Caravati, M. Bernasconi, T. D. Kühne, M. Krack, and M. Parrinello, Appl. Phys. Lett. 91, 171906 (2007) have also noted the importance of tetrahedral Ge sites; J. Hegedüs and S. R. Elliott, Nature Mater. 7, 399 (2008) have shown the importance of $A B A B$ units in the crystallization of $a$-GST.

${ }^{6}$ A. V. Kolobov, P. Fons, A. I. Frenkel, A. L. Ankudinov, J. Tominaga, and T. Uruga, Nature Mater. 3, 703 (2004), and references therein.

${ }^{7}$ C. Lang, S. A. Song, D. N. Manh, and D. J. H. Cockayne, Phys. Rev. B 76, 054101 (2007).

${ }^{8}$ D. A. Baker, M. A. Paesler, G. Lucovsky, S. C. Agarwal, and P. C. Taylor, Phys. Rev. Lett. 96, 255501 (2006).

${ }^{9}$ P. Jóvári, I. Kaban, J. Steiner, B. Beuneu, A. Schöps, and A. Webb, J. Phys.: Condens. Matter 19, 335212 (2007).

${ }^{10}$ J.-J. Kim, K. Kobayashi, E. Ikenaga, M. Kobata, S. Ueda, T. Matsunaga, K. Kifune, R. Kojima, and N. Yamada, Phys. Rev. B 76, 115124 (2007), this paper presents results for $a$ and $c$-alloys of the form $(\mathrm{GeTe})_{1-x}\left(\mathrm{Sb}_{2} \mathrm{Te}_{3}\right)_{x}$, including GST; see also $\mathrm{K}$. Kobayashi, M. Yabashi, Y. Takata, T. Tokushima, and S. Shin,
Appl. Phys. Lett. 83, 1005 (2003).

${ }^{11}$ See, for example, P. Biswas, R. Atta-Fynn, and D. A. Drabold, Phys. Rev. B 76, 125210 (2007); P. Biswas, D. N. Tafen, F. Inam, B. Cai, and D. A. Drabold, J. Phys.: Condens. Matter 21, 084207 (2009), and references therein.

${ }^{12}$ CPMD V3.12, Copyright IBM Corp 1990-2008, Copyright MPI für Festkörperforschung Stuttgart 1997-2001 (http:// www.cpmd.org).

${ }^{13}$ J. P. Perdew, K. Burke, and M. Ernzerhof, Phys. Rev. Lett. 77, 3865 (1996).

${ }^{14}$ J. Tao, J. P. Perdew, V. N. Staroverov, and G. E. Scuseria, Phys. Rev. Lett. 91, 146401 (2003).

${ }^{15}$ O. Gereben, P. Jóvári, L. Temleitner, and L. A. Pusztai, J. Optoelectron. Adv. Mater. 9, 3021 (2007).

${ }^{16}$ S. Kugler, K. Kohary, K. Kádas, and L. Pusztai, Solid State Commun. 127, 305 (2003).

${ }^{17}$ See EPAPS Document No. E-PRBMDO-80-R07926 for bond distances and bond angle distributions, partial coordination numbers, and frequently occurring Ge-centered configurations. $S(Q)$ for the DF optimized structure is also given. For more information on EPAPS, see http://www.aip.org/pubservs/epaps.html.

${ }^{18}$ Y. Fukuyama, N. Yasuda, J. Kim, H. Murayama, Y. Tanaka, S. Kimura, K. Kato, S. Kohara, Y. Moritomo, T. Matsunaga, R. Kojima, N. Yamada, H. Tanaka, T. Ohshima, and M. Takata, Appl. Phys. Express 1, 045001 (2008), and references therein.

${ }^{19}$ Fourfold coordinated Ge atoms are "tetrahedral" if the average deviation of the six bond angles (for bonds $\leq 3.0 \AA$ ) from the octahedral values $\left(90\right.$ and $180^{\circ}$ ) exceeds $10^{\circ}$.

${ }^{20}$ J. Harris, Phys. Rev. B 31, 1770 (1985). 